\title{
On unimodal maps with critical order $2+\varepsilon$
}

\author{
by
}

\section{Simin Li and Weixiao Shen (Hefei)}

\begin{abstract}
It is proved that a smooth unimodal interval map with critical order $2+\varepsilon$ has no wild attractor if $\varepsilon>0$ is small.
\end{abstract}

1. Introduction. In this paper, we continue the study of existence of wild attractors for unimodal interval maps. This problem, originated in [11], has been extensively studied. In particular, the original problem asked by Milnor, whether an S-unimodal interval map with a non-degenerate critical point has a wild attractor, was solved (see [9] and also [4, 5]). In [12], the second author of this paper extended the result to smooth unimodal maps with critical order not more than 2 . In this paper, we extend the result further, and prove that smooth unimodal maps with critical order not much larger than 2 have no wild attractor.

Note that there exist unimodal maps with wild attractors (see [1]). For definition of wild attractors and more historical remarks, see the references above.

Unimodal maps. Let $N=[a, b]$ be a compact interval. A $C^{1}$ map $f: N \rightarrow N$ is called unimodal if there exists a unique $c \in(a, b)$ (called the critical point) such that $f^{\prime}(c)=0$ and $f^{\prime}$ has different signs on the components of $N \backslash\{c\}$. Let $\mathcal{U}$ be the collection of unimodal maps $f$ which satisfy the following conditions:

- $f$ is $C^{3}$ except at $c$;

- there exists $\ell>1$ (called the critical order) and local $C^{3}$ diffeomorphisms $\phi$ and $\psi$ with $\phi(c)=0$ and $\psi(f(c))=0$ such that $\psi \circ f(x)=$ $-|\phi(x)|^{\ell}$ in a neighborhood of $c$. 
Note. Without loss of generality, we shall assume that the critical point of $f$ is located at 0 and $f(x)=-|x|^{\ell}+f(0)$ in a neighborhood of 0 .

Main Theorem. There exists $\varepsilon>0$ such that no $f \in \mathcal{U}$ with critical order $1<\ell \leq 2+\varepsilon$ has a wild attractor.

A map $f \in \mathcal{U}$ is called renormalizable if there exist an interval $I$ which contains the critical point $c$ in its interior, and a positive integer $s>1$, such that the intervals $I, f(I), \ldots, f^{s-1}(I)$ have pairwise disjoint interiors, $f^{s}(I) \subset I$, and $f^{s}(\partial I) \subset \partial I$. The unimodal map $f^{s}: I \rightarrow I$ is called a renormalization of $f$.

REMARK 1.1. It was already known to Milnor that if a map $f \in \mathcal{U}$ has a wild attractor, then $f$ is at most finitely renormalizable and the critical point $c$ is recurrent but not periodic. Since the property of having a wild attractor does not change under renormalization, we may restrict ourselves to the case that $f$ is non-renormalizable and has a recurrent and non-periodic critical point. Let $\mathcal{F}$ be the collection of all maps in $\mathcal{U}$ which satisfy these properties.

Recall that an open interval $T$ is called nice if $f^{n}(\partial T) \cap T=\emptyset$ for all $n \geq 0$. Let

$$
\mathrm{D}(T)=\left\{x \in N: f^{k}(x) \in T \text { for some } k \geq 1\right\} .
$$

The first entry map $R_{T}: \mathrm{D}(T) \rightarrow T$ is defined as $x \mapsto f^{k(x)}(x)$, where $k(x)$ is the entry time of $x$ into $T$, i.e., the minimal positive integer such that $f^{k(x)}(x) \in T$. It is well known that $k(x)$ is constant in any component of $\mathrm{D}(T)$. The map $\left.R_{T}\right|_{\mathrm{D}(T) \cap T}$ is called the first return map of $T$. A component of $\mathrm{D}(T)$ is called an entry domain, and a component of $\mathrm{D}(T) \cap T$ is called a return domain.

Principal nest. Consider $f \in \mathcal{F}$. Let $q$ denote the orientation-reversing fixed point of $f$. Let $I^{0}=(\hat{q}, q)$ where $f(\hat{q})=f(q)$, and for all $n \geq 1$, let $I^{n}$ be the return domain to $I^{n-1}$ which contains the critical point. All these intervals $I^{n}$ are nice. The sequence

$$
I^{0} \supset I^{1} \supset I^{2} \supset \cdots
$$

is called the principal nest. Let $g_{n}$ denote the first return map to $I^{n}$. Let $m(0)=0$, and let $m(1)<m(2)<\cdots$ be all the non-central return moments, i.e., positive integers such that

$$
g_{m(k)-1}(0) \notin I^{m(k)} .
$$

Note that the fact that $f$ is non-renormalizable implies that there are infinitely many non-central return moments. 
Main Lemma. For any $\eta>0$ there exists $\varepsilon>0$ such that for $f \in \mathcal{F}$ with critical order $1<\ell \leq 2+\varepsilon$, we have

$$
\limsup _{k \rightarrow \infty} \frac{\left|I^{m(k)+1}\right|}{\left|I^{m(k)}\right|}<\eta
$$

This result was claimed in [12] without a detailed proof. The proof given here is a modification of the method in that paper.

In [2], the authors assume this lemma, and prove that $f \in \mathcal{F}$ with critical order $2+\varepsilon$ has an acip provided that $\varepsilon$ is small and $f$ has only finitely many central returns, i.e., $m(k)-k$ is constant for all large $k$.

The main theorem and the main lemma also extend some results of [6].

Proposition 1.1. For any $\ell_{0}>1$ there exists $\alpha=\alpha\left(\ell_{0}\right)>0$ with the following property. Let $f \in \mathcal{F}$ be a map with critical order $1<\ell \leq \ell_{0}$ and assume that $\lim \sup \left|I^{m(k)+1}\right| /\left|I^{m(k)}\right|<\alpha$. Then $f$ has no wild attractor.

This proposition was (implicitly) proved in Section 7 of [9] in the case that $f$ has negative Schwarzian. The last assumption becomes unnecessary due to [7]. One can also prove this proposition using the Theorem of [3] or the Theorem of [8].

Deduction of the Main Theorem from the Main Lemma. Let $\alpha=\alpha(3)$ be as in Proposition 1.1. By the Main Lemma, there exists $\varepsilon \in(0,1)$ such that if $f \in \mathcal{F}$ has critical order $\ell \in(1,2+\varepsilon)$ then the assumption of Proposition 1.1 is satisfied, so $f$ has no wild attractor. By the remark above, it follows that no map $f \in \mathcal{U}$ with critical order $\ell \in(1,2+\varepsilon)$ has a wild attractor.

1.1. Preliminaries. The following two lemmas were proved in [12].

LeMma 1.2. Let $J \subset I^{m(k)-1}-I^{m(k)}$ be a return domain to $I^{m(k)-1}$ with return time s. Then there is an interval $J^{\prime}$ with $J \subset J^{\prime} \subset I^{m(k)-1}-I^{m(k)}$ such that $f^{s}: J^{\prime} \rightarrow I^{m(k-1)}$ is a diffeomorphism.

LEMMA 1.3. Let $s$ be the return time of 0 to $I^{m(k)}$. Then there is an interval $J \ni f(0)$ with $f^{-1}(J) \subset I^{m(k)}$ such that $f^{s-1}: J \rightarrow I^{m(k-1)}$ is a diffeomorphism.

The following lemma on real bounds was proved in [10, 7].

Lemma 1.4. For any $\ell_{0}>1$, there exists a constant $\varrho>1$ such that if $f \in \mathcal{F}$ has critical order $\ell \in\left(1, \ell_{0}\right)$, then for all $k$ sufficiently large,

$$
\left|I^{m(k)}\right| \geq \varrho\left|I^{m(k)+1}\right| .
$$

Moreover, if $g_{m(k)}\left(I^{m(k)+1}\right) \not \supset 0$, then

$$
\left|I^{m(k+1)-1}\right| \geq \varrho\left|I^{m(k+1)}\right| .
$$


We shall use the following cross-ratio. For any two intervals $J \Subset T$, we define

$$
C(T, J)=\frac{|T||J|}{|L||R|}
$$

where $L, R$ are the components of $T-J$. If $h: T \rightarrow \mathbb{R}$ is a homeomorphism onto its image, we write

$$
\mathbf{C}(h ; T, J)=\frac{C(h(T), h(J))}{C(T, J)} .
$$

Lemma 1.5 ([7, Theorem C]). For each $k$ sufficiently large, there is a positive number $\mathcal{O}_{k}$ with $\mathcal{O}_{k} \rightarrow 1$ as $k \rightarrow \infty$ and with the following property. Let $T \subset[-1,1]$ be an interval and let $n$ be a positive integer. Assume that $\left.f^{n}\right|_{T}$ is monotone and $f^{n}(T) \subset I^{m(k-1)}$. Then for any interval $J \Subset T$, we have

$$
\mathbf{C}\left(f^{n} ; T, J\right) \geq \mathcal{O}_{k} .
$$

We shall also use the following lemma which is implicit in the proof of the Main Theorem of [12, p. 390].

Lemma 1.6. For any $\ell_{0}>1$, there is a constant $C=C\left(\ell_{0}\right)>0$ such that for any $f \in \mathcal{F}$ with critical order $1<\ell \leq \ell_{0}$ and sufficiently large $k \geq 1$, we have

$$
\frac{\left|I^{m(k)}\right|}{\left|I^{m(k+1)}\right|} \geq \frac{\left|I^{m(k)}\right|}{\left|I^{m(k)+1}\right|} \geq C\left(\frac{\left|I^{m(k-1)}\right|}{\left|I^{m(k)}\right|}\right)^{1 / \ell} .
$$

2. Lower limit. For any $n \geq 0$, write $c_{n}=f^{n}(0)$. A closest (critical) return time is a positive integer $s$ such that $c_{k} \notin\left(c_{s},-c_{s}\right)$ for all $1 \leq k \leq s$. The point $c_{s}$ will be called a closest (critical) return.

Let $s_{1}<s_{2}<\cdots$ be all the closest return times. Let $n_{0}$ be such that $s_{n_{0}}$ is the return time of 0 to $I^{m(1)}$. For any $n \geq n_{0}$, let $k=k(n)$ be so that $c_{s_{n}} \in I^{m(k)}-I^{m(k+1)}$. Note that $c_{s_{n}} \in I^{m(k+1)-1}-I^{m(k+1)}$. Let $T_{n} \ni c_{s_{n}}$ be the maximal open interval such that:

- $\left.f^{s_{n+1}-s_{n}}\right|_{T_{n}}$ is monotone,

- $f^{s_{n+1}-s_{n}}\left(T_{n}\right) \subset I^{m(k)-1}$.

Let $x_{n}, y_{n}$ denote the endpoints of $f^{s_{n+1}-s_{n}}\left(T_{n}\right)$, with $\left|x_{n}\right| \leq\left|y_{n}\right|$. By Lemma 3.2 of [12], $y_{n} \in \partial I^{m(k)-1}, x_{n} \notin I^{m(k)}$, and $\left(x_{n}, y_{n}\right) \ni 0$. Let $b_{n}$ be an endpoint of $I^{m(k+1)-1}$. Define

$$
A_{n}=\frac{\left|b_{n}\right|^{\ell}-\left|c_{s_{n+1}}\right|^{\ell}}{\left|b_{n}\right|^{\ell}-\left|c_{s_{n}}\right|^{\ell}}, \quad B_{n}=\left(\frac{\left|c_{s_{n}}\right|}{\left|c_{s_{n+1}}\right|}\right)^{\ell / 2},
$$

and

$$
V_{n}=\frac{2\left|x_{n}\right|\left(\left|y_{n}\right|+\left|c_{s_{n}}\right|\right)}{\left(\left|y_{n}\right|+\left|x_{n}\right|\right)\left(\left|x_{n}\right|+\left|c_{s_{n}}\right|\right)}, \quad W_{n}=\left(\frac{\left|x_{n}\right|}{\left|c_{s_{n-1}}\right|}\right)^{\ell / 2} .
$$


Moreover, define

$$
\widetilde{A}_{n}=\frac{\left|b_{n}\right|^{2}-\left|c_{s_{n+1}}\right|^{2}}{\left|b_{n}\right|^{2}-\left|c_{s_{n}}\right|^{2}}, \quad \widetilde{W}_{n}=\frac{\left|x_{n}\right|}{\left|c_{s_{n-1}}\right|} .
$$

The argument in [12] shows the following

Proposition 2.1. There exists a constant $\sigma_{0}>1$ such that if $\ell<3$ then for all $n$ sufficiently large, we have

$$
\widetilde{A}_{n-1} V_{n} \widetilde{W}_{n} \geq \sigma_{0}
$$

Let

$$
\mu_{k}=\frac{\left|I^{m(k+1)}\right|}{\left|I^{m(k)}\right|} .
$$

Proposition 2.2. There is a constant $\sigma>1$ such that for any $\alpha>0$, there exists $\varepsilon=\varepsilon(\alpha)>0$ which satisfies the following. If $f \in \mathcal{F}$ has critical order $1<\ell \leq 2+\varepsilon$ and $n$ is sufficiently large and $\mu_{k-1}, \mu_{k}, \mu_{k+1} \geq \alpha$, where $k=k(n)$, then

$$
\begin{aligned}
& \frac{\left|\left(f^{s_{n+1}}\right)^{\prime}\left(c_{1}\right)\right| B_{n} A_{n-1}}{\left|\left(f^{s_{n}}\right)^{\prime}\left(c_{1}\right)\right| B_{n-1} A_{n}} \geq \sigma, \\
& \frac{\left|\left(f^{s_{n+1}}\right)^{\prime}\left(c_{1}\right)\right| B_{n}}{\left|\left(f^{s_{n}}\right)^{\prime}\left(c_{1}\right)\right| B_{n-1}} \geq \frac{1}{2} A_{n} V_{n} W_{n} .
\end{aligned}
$$

Proof. By the Main Lemma of [12], we only need to consider the case $\ell>2$. Given $\alpha>0$. Suppose $f \in \mathcal{F}$ has critical order $\ell=2+\varepsilon$ for some $\varepsilon>0$. By the definition, $c_{s_{n-1}} \in I^{m(k-1)}-I^{m(k+1)}, c_{s_{n+1}} \in I^{m(k)}-I^{m(k+2)}$ and $x_{n}, y_{n} \in \overline{I^{m(k)-1}}-I^{m(k)}$.

By the Lemma in [12], $\phi(x, \ell)=x^{1-\ell / 2} \int_{x}^{1} t^{\ell-1} d t$ is increasing in $\ell$. Now the computation of $[12$, p. 397 , first paragraph] yields

$$
\left|\left(f^{s_{n+1}-s_{n}}\right)^{\prime}\left(f\left(c_{s_{n}}\right)\right)\right| \frac{A_{n-1} B_{n}}{A_{n} B_{n-1}} \geq g\left(\frac{\left|c_{s_{n+1}}\right|}{\left|x_{n}\right|}, \varepsilon\right) \mathcal{O}_{k} A_{n-1} V_{n} W_{n},
$$

where

$$
g(t, \varepsilon)=\frac{\phi(t, 2)}{\phi(t, 2+\varepsilon)} .
$$

Since

$$
\frac{\left|c_{s_{n+1}}\right|}{\left|x_{n}\right|} \geq \frac{\left|I^{m(k+2)}\right|}{\left|I^{m(k-1)}\right|} \geq \alpha^{3}
$$

for any $0<\sigma_{1}<1$, if $\varepsilon$ is small enough,

$$
g\left(\frac{\left|c_{s_{n+1}}\right|}{\left|x_{n}\right|}, \varepsilon\right)>\sigma_{1} \text {. }
$$

In particular, (2.2) holds. 
Let us prove (2.1). Since

$$
\widetilde{W}_{n} \geq \frac{\left|I^{m(k)}\right|}{\left|I^{m(k-1)}\right|} \geq \alpha,
$$

for any $0<\sigma_{2}<1$, provided that $\varepsilon$ is small enough,

$$
W_{n}=\widetilde{W}_{n}^{\varepsilon / 2} \widetilde{W_{n}} \geq \sigma_{2} \widetilde{W}_{n} .
$$

Since $\left|c_{s_{n-1}}\right| /\left|y_{n}\right| \geq\left|I^{m(k+1)}\right| /\left|I^{m(k-1)}\right| \geq \alpha^{2}$ and $\left|b_{n-1}\right|=\left|y_{n}\right|$, we have

$$
A_{n-1}=\frac{\left|y_{n}\right|^{2+\varepsilon}-\left|c_{s_{n}}\right|^{2+\varepsilon}}{\left|y_{n}\right|^{2+\varepsilon}-\left|c_{s_{n-1}}\right|^{2+\varepsilon}} \geq \frac{1-\left(\frac{\left|c_{s_{n}}\right|}{\left|y_{n}\right|}\right)^{2}}{1-\left(\frac{\left|c_{s_{n-1}}\right|}{\left|y_{n}\right|}\right)^{2}\left(\frac{\left|s_{s_{n-1}}\right|}{\left|y_{n}\right|}\right)^{\varepsilon}} \geq \sigma_{3} \widetilde{A}_{n-1}
$$

where $\sigma_{3}>0$ can be arbitrarily close to 1 if $\varepsilon$ is small.

Therefore we get

$$
\left|\left(f^{s_{n+1}-s_{n}}\right)^{\prime}\left(f\left(c_{s_{n}}\right)\right)\right| \frac{A_{n-1} B_{n}}{A_{n} B_{n-1}} \geq \sigma_{1} \sigma_{2} \sigma_{3} \mathcal{O}_{k} \widetilde{A}_{n-1} V_{n} \widetilde{W}_{n}
$$

where $\sigma_{1}, \sigma_{2}, \sigma_{3}$ can be arbitrarily close to 1 if $\varepsilon$ is sufficiently small. By Proposition 2.1, (2.1) follows.

Corollary 2.3. For any $\alpha>0$, there exists $\varepsilon=\varepsilon(\alpha)>0$ such that for $f \in \mathcal{F}$ with critical order $1<\ell \leq 2+\varepsilon$, we have

$$
\liminf _{k \rightarrow \infty} \frac{\left|I^{m(k+1)}\right|}{\left|I^{m(k)}\right|}<\alpha
$$

Proof. Suppose the statement is not true. Then there exists $\alpha>0$, and for any $\varepsilon>0$ there exists a map $f \in \mathcal{F}$ with critical order $1<\ell \leq 2+\varepsilon$ such that

$$
\liminf _{k \rightarrow \infty} \frac{\left|I^{m(k+1)}\right|}{\left|I^{m(k)}\right|} \geq \alpha
$$

Therefore for $k$ large enough, $\left|I^{m(k+1)}\right| /\left|I^{m(k)}\right| \geq \alpha / 2$. Provided that $\varepsilon$ is small enough, Proposition 2.2 implies that

$$
\left|\left(f^{s_{n+1}-s_{n}}\right)^{\prime}\left(f\left(c_{s_{n}}\right)\right)\right| \frac{A_{n-1} B_{n}}{A_{n} B_{n-1}}>\sigma
$$

for all $n$ large, where $\sigma>1$ is a constant. But as shown in the proof of the Main Theorem in [12], this implies that $f$ has decay of geometry and thus $\lim \left|I^{m(k+1)}\right| /\left|I^{m(k)}\right|=0$, which is a contradiction.

\section{Proof of the Main Lemma}

Proposition 3.1. For any $\ell_{0}>1$, there exists a constant $C=C\left(\ell_{0}\right)$ $>0$ satisfying the following. Let $f \in \mathcal{F}$ be a map with critical order $1<\ell<\ell_{0}$. Assume that $k$ is sufficiently large and $\mu_{k}, \mu_{k-1}<\alpha$. Let $n$ be such that $s_{n}$ 
is the return time of 0 into $I^{m(k)}$. Then

$$
\left|\left(f^{s_{n}}\right)^{\prime}\left(c_{1}\right)\right| B_{n-1}>C / \sqrt{\alpha} \text {. }
$$

Proof. Notice that $c_{s_{n}} \in I^{m(k+1)-1}-I^{m(k+1)}$ is also the first return of 0 to $I^{m(k+1)-1}$. By Lemma 1.3 , there is an interval $J \ni c_{1}$ such that $f^{-1}(J) \subset I^{m(k)}$ and $f^{s_{n}-1}: J \rightarrow I^{m(k-1)}$ is a diffeomorphism. We will consider two cases.

CASE 1: $\left|f^{s_{n}}\left(I^{m(k+1)}\right)\right| \geq 0.1\left|I^{m(k+1)-1}\right|$. Since $f\left(I^{m(k+1)}\right) \subset J$ and $f^{s_{n}}\left(I^{m(k+1)}\right) \subset I^{m(k)}$ is well-inside $I^{m(k-1)},\left.f^{s_{n}-1}\right|_{f\left(I^{m(k+1)}\right)}$ has uniformly bounded distortion. Therefore

$$
\left|\left(f^{s_{n}-1}\right)^{\prime}\left(c_{1}\right)\right| \geq C \frac{\left|f^{s_{n}}\left(I^{m(k+1)}\right)\right|}{\left|f\left(I^{m(k+1)}\right)\right|} \geq C \frac{\left|I^{m(k+1)-1}\right|}{\left|I^{m(k+1)}\right|^{\ell}} .
$$

So

$$
\begin{aligned}
\left|\left(f^{s_{n}}\right)^{\prime}\left(c_{1}\right)\right| B_{n-1} & =\left|\left(f^{s_{n}-1}\right)^{\prime}\left(c_{1}\right)\right|\left|f^{\prime}\left(c_{s_{n}}\right)\right|\left(\frac{\left|c_{s_{n-1}}\right|}{\left|c_{s_{n}}\right|}\right)^{\ell / 2} \\
& \geq C \frac{\left|I^{m(k+1)-1}\right|}{\left|I^{m(k+1)}\right| \ell} \ell\left|I^{m(k+1)}\right|^{\ell-1}\left(\frac{\left|I^{m(k)}\right|}{\left|I^{m(k+1)-1}\right|}\right)^{\ell / 2} \\
& \geq C \frac{\left|I^{m(k+1)-1}\right|}{\left|I^{m(k+1)}\right|}\left(\frac{\left|I^{m(k)}\right|}{\left|I^{m(k+1)-1}\right|}\right)^{\ell / 2} .
\end{aligned}
$$

If $m(k+1)=m(k)+1$, then

$$
\left|\left(f^{s_{n}}\right)^{\prime}\left(c_{1}\right)\right| B_{n-1} \geq C \frac{\left|I^{m(k)}\right|}{\left|I^{m(k+1)}\right|} \geq \frac{C}{\alpha} .
$$

If $m(k+1) \geq m(k)+2$, then

$$
\left|\left(f^{s_{n}}\right)^{\prime}\left(c_{1}\right)\right| B_{n-1} \geq C\left(\frac{\left|I^{m(k)}\right|}{\left|I^{m(k+1)-1}\right|}\right)^{\ell / 2} \geq C\left(\frac{\left|I^{m(k)}\right|}{\left|I^{m(k)+1}\right|}\right)^{\ell / 2} \geq \frac{C}{\sqrt{\alpha}}
$$

by Lemma 1.6.

CASE 2: $\left|f^{s_{n}}\left(I^{m(k+1)}\right)\right|<0.1\left|I^{m(k+1)-1}\right|$. First we assume that $m(k+1)$ $=m(k)+1$. By Lemma 1.4, $\varrho^{\prime}=\left|I^{m(k-1)}\right| /\left|I^{m(k)}\right|>\varrho>1$. Let $J^{\prime} \subset J$ be such that $f^{s_{n}-1}\left(J^{\prime}\right)=\frac{\varrho^{\prime}+1}{2} I^{m(k)}$. Let $K^{\prime}=f^{-1}\left(J^{\prime}\right) \subset I^{m(k)}$ and $K$ be a component of $K^{\prime}-\{0\}$.

Since $\frac{\varrho^{\prime}+1}{2} I^{m(k)}$ is well-inside $I^{m(k+1)},\left.f^{s_{n}-1}\right|_{J^{\prime}}$ has uniformly bounded distortion. Thus

$$
\left|\left(f^{s_{n}-1}\right)^{\prime}\left(c_{1}\right)\right| \geq C \frac{\left|f^{s_{n}} K\right|}{|f(K)|} \geq C \frac{\left|I^{m(k-1)}\right|}{\left|I^{m(k)}\right| \ell} .
$$


By assumption, $c_{s_{n}}$ is close to the endpoint of $I^{m(k)}$, and it follows that

$$
\begin{aligned}
\left|\left(f^{s_{n}}\right)^{\prime}\left(c_{1}\right)\right| B_{n-1} & \geq\left|\left(f^{s_{n}}\right)^{\prime}\left(c_{1}\right)\right|=\left|\left(f^{s_{n}-1}\right)^{\prime}\left(c_{1}\right)\right|\left|f^{\prime}\left(c_{s_{n}}\right)\right| \\
& \geq C \frac{\left|I^{m(k-1)}\right|}{\left|I^{m(k)}\right|^{\ell}}\left|I^{m(k)}\right|^{\ell-1} \geq \frac{C}{\alpha} .
\end{aligned}
$$

Now we assume that $m(k+1)-m(k) \geq 2$. By Lemma 1.4, $\varrho^{\prime}:=$ $\left|I^{m(k)}\right| /\left|I^{m(k+1)-1}\right| \geq\left|I^{m(k)}\right| /\left|I^{m(k)+1}\right|>\varrho$. Let $J^{\prime} \subset J$ be such that $f^{s_{n}-1}\left(J^{\prime}\right)$ $=T:=\frac{\varrho^{\prime}+1}{2} I^{m(k+1)-1}$. Let $K^{\prime}=f^{-1}\left(J^{\prime}\right)$. Then $K^{\prime} \subset T$, since otherwise $f^{s_{n}}$ will have an attracting periodic point in $K^{\prime}$. Let $K$ be a component of $K^{\prime}-\{0\}$. Since $\left.f^{s_{n}-1}\right|_{J^{\prime}}$ has uniformly bounded distortion, we have

$$
\left|\left(f^{s_{n}-1}\right)^{\prime}\left(c_{1}\right)\right| \geq C \frac{\left|f^{s_{n}}(K)\right|}{|f(K)|} \geq C \frac{\left|I^{m(k+1)-1}\right|}{\mid I^{m(k+1)-1 \mid \ell}} .
$$

By assumption, $c_{s_{n}}$ is close to $\partial I^{m(k+1)-1}$, and it follows that $\left|\left(f^{s_{n}}\right)^{\prime}\left(c_{1}\right)\right|=$ $\left|\left(f^{s_{n}-1}\right)^{\prime}\left(c_{1}\right)\right|\left|f^{\prime}\left(c_{s_{n}}\right)\right|$ is bounded away from 0 . Therefore

$$
\left|\left(f^{s_{n}}\right)^{\prime}\left(c_{1}\right)\right| B_{n-1} \geq C\left(\frac{\left|c_{s_{n-1}}\right|}{\left|c_{s_{n}}\right|}\right)^{\ell / 2} \geq C\left(\frac{\left|I^{m(k)}\right|}{\left|I^{m(k+1)-1}\right|}\right)^{\ell / 2} \geq \frac{C}{\sqrt{\alpha}},
$$

where we use Lemma 1.6.

Lemma 3.2. There is a constant $\beta>0$ such that $W_{n} \geq \beta$ for each $n>0$.

Proof. For each $n>0$ let $k>0$ be such that $c_{s_{n}} \in I^{m(k)}-I^{m(k+1)}$ and let $p=m(k+1)-m(k)$. We may assume that $W_{n}<1$, so by Lemma 3.2 of [12], we have $p \geq 2,0 \notin g_{m(k)}\left(I^{m(k)+1}\right)$ and $c_{s_{n}}$ is the first return of 0 to $I^{m(k)}$. Moreover, let $q$ be such that $c_{s_{n}}=g_{m(k)-1}^{q}(0)$. Then there exist $1 \leq q^{\prime}<q$ and $1 \leq p^{\prime}<p$ such that $x_{n}=g_{m(k)-1}^{q^{\prime}}\left(g_{m(k)}^{p^{\prime}}(0)\right)$ and $c_{s_{n-1}}=g_{m(k)-1}^{q^{\prime}}(0)$.

Let $J_{1}$ be the entry domain to $I^{m(k)}$ which contains $c_{s_{n-1}}=g_{m(k)-1}^{q^{\prime}}(0)$. Then $\left.g_{m(k)-1}^{q-q^{\prime}}\right|_{J_{1}}: J_{1} \rightarrow I^{m(k)}$ is a diffeomorphism and

$$
I^{m(k)-1}-I^{m(k)} \supset J_{1} \supset g_{m(k)-1}^{q^{\prime}}\left(I^{m(k)+1}\right) \ni g_{m(k)-1}^{q^{\prime}}\left(g_{m(k)}^{p^{\prime}}(0)\right)=x_{n} .
$$

Therefore $g_{m(k)-1}^{q-q^{\prime}}\left(\left(x_{n}, c_{s_{n-1}}\right)\right) \subset\left(c_{s_{n}}, w_{n}\right)$, where $w_{n}=g_{m(k)}^{p}(0) \in I^{m(k)}$ $-I^{m(k)+1}$.

Let $J \subset I^{m(k)-1}-I^{m(k)}$ be the entry domain to $I^{m(k)-1}$ which contains $c_{s_{n-1}}$. Then $J \supset J_{1}$. By Lemma 1.2, there is an interval $J^{\prime}$ with $J \subset J^{\prime}$ $\subset I^{m(k)-1}-I^{m(k)}$ such that $g_{m(k)-1}^{q-q^{\prime}}: J^{\prime} \rightarrow I^{m(k-1)}$ is a diffeomorphism. Since $\left(c_{s_{n}}, w_{n}\right)$ is well-inside $I^{m(k-1)},\left(x_{n}, c_{s_{n-1}}\right)$ is well-inside $J^{\prime}$. Since $0 \notin J^{\prime}$, it follows that $W_{n}$ is bounded away from zero. 
Before proving the Main Lemma we need a lemma which is implicit in the proof of the Main Theorem in [12].

Lemma 3.3. For any $\ell_{0}>1$ and $\eta>0$, there exists $\xi>0$ satisfying the following. Let $f \in \mathcal{F}$ be a map with critical order $1<\ell \leq \ell_{0}$, and assume that $\left|\left(f^{s_{n}}\right)^{\prime}\left(c_{1}\right)\right| B_{n-1}>\xi$ for all sufficiently large $n$. Then

$$
\frac{\left|I^{m(k)+1}\right|}{\left|I^{m(k)}\right|}<\eta
$$

for all sufficiently large $k$.

Proof of the Main Lemma. For any $\alpha>0$, by Lemma 1.6, there exists $\alpha_{1}>0$ (we may assume $\alpha_{1}<\alpha$ ) such that if $\mu_{k-1}<\alpha_{1}$, then $\mu_{k}<\alpha$. By Corollary 2.3, there exists $\varepsilon>0$ such that if $f$ is a non-renormalizable $C^{3}$ unimodal map with critical order $\ell \leq 2+\varepsilon$, then

$$
\liminf _{k \rightarrow \infty} \frac{\left|I^{m(k+1)}\right|}{\left|I^{m(k)}\right|}<\alpha_{1}
$$

Let $k_{1}<k_{2}<\cdots$ be all the integers such that $\mu_{k_{i}-1}<\alpha_{1}$. Then $\mu_{k_{i}}<\alpha$ and $\mu_{k} \geq \alpha_{1}$ if $k \neq k_{i}-1(i=1,2, \ldots)$.

To complete the proof, by Lemma 3.3, it is enough to prove that the quantity $\left|\left(f^{s_{m}}\right)^{\prime}\left(c_{1}\right)\right| B_{m-1}$ is large for $m$ sufficiently large.

Fix a large integer $i \geq 1$. Let $n=n(i)$ be such that $s_{n}$ is the return time of 0 into $I^{m\left(k_{i}\right)}$. Since $\mu_{k_{i}-1}, \mu_{k_{i}}<\alpha$, by Proposition 3.1,

$$
\left|\left(f^{s_{n}}\right)^{\prime}\left(c_{1}\right)\right| B_{n-1}>C / \sqrt{\alpha}
$$

for some constant $C>0$.

Certainly we may assume that $k_{i+1} \geq k_{i}+2$, so that $\mu_{k} \geq \alpha_{1}$ for all $k_{i} \leq k \leq k_{i+1}-2$. By Lemma 1.6, there exists $\alpha_{2} \in\left(0, \alpha_{1}\right)$ such that $\mu_{k_{i}-1} \geq \alpha_{2}$. Let $N=N(i)$ be such that $c_{s_{n+N}}$ is the first return of 0 to $I^{m\left(k_{i+1}-1\right)}$. By Proposition 2.2, for any $n \leq m \leq n+N-1$,

$$
\frac{\left|\left(f^{s_{m+1}}\right)^{\prime}\left(c_{1}\right)\right| B_{m}}{\left|\left(f^{s_{m}}\right)^{\prime}\left(c_{1}\right)\right| B_{m-1}} \geq \frac{1}{2} A_{m} V_{m} W_{m}
$$

If $\left|\left(f^{s_{m+1}}\right)^{\prime}\left(c_{1}\right)\right| B_{m} \geq\left|\left(f^{s_{m}}\right)^{\prime}\left(c_{1}\right)\right| B_{m-1}$ for all $n \leq m \leq n+N-1$, then $\left|\left(f^{s_{m}}\right)^{\prime}\left(c_{1}\right)\right| B_{m-1}>C / \sqrt{\alpha}$ for all $n \leq m \leq n+N$. Otherwise, let $n+N-1 \geq$ $\widetilde{n} \geq n$ be minimal such that

$$
\left|\left(f^{s_{\tilde{n}+1}}\right)^{\prime}\left(c_{1}\right)\right| B_{\widetilde{n}}<\left|\left(f^{s_{\tilde{n}}}\right)^{\prime}\left(c_{1}\right)\right| B_{\widetilde{n}-1} .
$$

Then $A_{\widetilde{n}} V_{\widetilde{n}} W_{\widetilde{n}} \leq 2$. Since $V_{\widetilde{n}}>1$ and $W_{\widetilde{n}} \geq \beta$ (Lemma 3.2), we obtain $A_{\widetilde{n}} \leq 1 / 2 \beta$. Since

$$
\left|\left(f^{s_{\tilde{n}}}\right)^{\prime}\left(c_{1}\right)\right| B_{\tilde{n}-1} \geq \cdots \geq\left|\left(f^{s_{n}}\right)^{\prime}\left(c_{1}\right)\right| B_{n-1} \geq C / \sqrt{\alpha},
$$


and

we have

$$
\frac{\left|\left(f^{s_{\tilde{n}+1}}\right)^{\prime}\left(c_{1}\right)\right| B_{\widetilde{n}}}{\left|\left(f^{s_{\tilde{n}}}\right)^{\prime}\left(c_{1}\right)\right| B_{\widetilde{n}-1}} \geq \frac{1}{2} A_{\widetilde{n}} V_{\widetilde{n}} W_{\widetilde{n}} \geq \frac{\beta}{2}
$$

$$
\left|\left(f^{s_{\widetilde{n}+1}}\right)^{\prime}\left(c_{1}\right)\right| \frac{B_{\widetilde{n}}}{A_{\widetilde{n}}} \geq \frac{\beta^{2} C}{\sqrt{\alpha}} .
$$

Now by Proposition 2.2, for any $\tilde{n}+1 \leq m \leq n+N$,

$$
\left|\left(f^{s_{m}}\right)^{\prime}\left(c_{1}\right)\right| B_{m-1} \geq\left|\left(f^{s_{m}}\right)^{\prime}\left(c_{1}\right)\right| \frac{B_{m-1}}{A_{m-1}}>\frac{\beta^{2} C}{\sqrt{\alpha}} .
$$

Since $\alpha>0$ can be arbitrarily small, the proof is finished.

\section{References}

[1] H. Bruin, G. Keller, T. Nowicki and S. van Strien, Wild Cantor attractors exist, Ann. of Math. (2) 143 (1996), 97-130.

[2] H. Bruin, W. X. Shen and S. van Strien, Invariant measures exist without a growth condition, Comm. Math. Phys. 241 (2003), 287-306.

[3] - - - - - Existence of SRB measure is typical for unimodal polynomial families, preprint, 2004.

[4] J. Graczyk, D. Sands and G. Świątek, Decay of geometry for unimodal maps: Negative Schwarzian case, Ann. of Math. (2) 161 (2005), 613-677.

[5] - - - -, Metric attractors for smooth unimodal maps, ibid. 159 (2004), 725-740.

[6] G. Keller and T. Nowicki, Fibonacci maps re(al)visited, Ergodic Theory Dynam. Systems 15 (1995), 99-120.

[7] O. Kozlovski, How to get rid of the negative Schwarzian condition, Ann. of Math. (2) 152 (2000), 743-762.

[8] S. M. Li and W. X. Shen, Hausdorff dimension of Cantor attractors in one-dimensional dynamics, preprint, 2005.

[9] M. Lyubich, Combinatorics, geometry and attractors of quasi-quadratic maps, Ann. of Math. (2) 140 (1994), 347-404.

[10] M. Martens, Distortion results and invariant Cantor sets of unimodal maps, Ergodic Theory Dynam. Systems 14 (1994), 331-349.

[11] J. Milnor, On the concept of attractors, Comm. Math. Phys. 99 (1985), 177-195, and 102 (1985), 517-519.

[12] W. X. Shen, Decay geometry for unimodal maps: an elementary proof, Ann. of Math. (2) 163 (2006), 383-404.

Mathematics Department

University of Science and Technology of China

Hefei, 230026, China

E-mail: lsm@ustc.edu.cn wxshen@ustc.edu.cn 